\title{
Preconditioning cubic collocation method for elliptic equations
}

\author{
Sang Hun LeE and Byeong Chun SHIN
}

(Received June 17, 1996)

\begin{abstract}
In this paper we provide the preconditioning results of the linear system generated by the cubic spline collocation discretization with penalty terms for an elliptic equation with Neumann boundary conditions. Moreover, we show that the linear system of an elliptic equation with Dirichlet or mixed boundary conditions can be directly derived from the linear system of the same equation with Neumann boundary conditions.
\end{abstract}

Key words: $\mathrm{C}^{1}$-cubic spline, preconditioning collocation method.

\section{Introduction}

In this paper, we will provide preconditioning results of the cubic spline collocation discretization with penalty terms for a positive definite second order elliptic boundary value problem with Neumann boundary conditions.

In the course of patching collocation method introduced by Orszag in [O] for elliptic boundary value problems, Funaro has provided a method to handle different types of boundary conditions in [F2]. The idea is to collocate an equation at both the interior nodes and the boundary nodes of a given interval for the equations to be solved at the boundary points. In this paper, we will deal with the preconditioning cubic spline collocation method for the Neumann problem following the ideas provided in [F2] and [KP].

Let us consider an equation

$$
-u^{\prime \prime}+c u=f \quad \text { in }(0,1)
$$

with the Neumann boundary conditions

$$
u^{\prime}(0)=0, \quad u^{\prime}(1)=0,
$$

1991 Mathematics Subject Classification : 65N30, 65N35.

This paper was supported by Korean Ministry of Education through Research Fund (BSRI-96-1401) and TGRC-KOSEF. 
where $f$ and $c$ are continuous functions and

$$
0<m \leq c(x) \leq M<\infty \text { for } 0 \leq x \leq 1 .
$$

Let us introduce the space $\mathcal{S}_{\Delta, 3}$ of $C^{1}$-cubic spline functions with $C^{1}$-cubic Lagrange spline basis $\left\{\psi_{i}\right\}_{i=0}^{2 N+1}$ with respect to a given partition $\Delta:=$ $\left\{t_{i}\right\}_{i=0}^{N}$ of the unit interval $I$ where $\psi_{i}\left(\xi_{j}\right)=\delta_{i j}$ for given collocation points $\left\{\xi_{j}\right\}_{j=0}^{2 N+1}$ such that, for $j=1,2, \ldots, N$,

$$
\xi_{0}=0, \quad t_{j-1}<\xi_{2 j-1}<\xi_{2 j}<t_{j}, \quad \xi_{2 N+1}=1 .
$$

Notice that $\left\{\psi_{i}\right\}_{i=1}^{2 N}$ can be chosen from [KK] or [KS] in the sense that $\psi_{i}$ satisfies the Dirichlet boundary conditions, i.e., $\psi_{i}(0)=0$ and $\psi_{i}(1)=0$, and in order to handle the Neumann boundary conditions $\psi_{0}$ and $\psi_{2 N+1}$ can be added by following the ideas in the proceeding references.

Concerning the numerical approximation, we look for $u \in \mathcal{S}_{\Delta, 3}$, and we collocate the Eq. (1.1) at the interior points $\xi_{j}$, i.e.,

$$
\left(-u^{\prime \prime}+c u\right)\left(\xi_{j}\right)=f\left(\xi_{j}\right), \quad j=1,2, \ldots, 2 N .
$$

For the equation to be solved at the boundary points 0 and 1, we will impose the following conditions as penalty terms:

$$
\begin{aligned}
\left(-u^{\prime \prime}+c u\right)(0)-w_{0}^{-1} u^{\prime}(0) & =f(0), \\
\left(-u^{\prime \prime}+c u\right)(1)+w_{2 N+1}^{-1} u^{\prime}(1) & =f(1)
\end{aligned}
$$

where $\left\{w_{i}\right\}_{i=0}^{2 N+1}$ are the quadrature weights relative to the points $\left\{\xi_{i}\right\}_{i=0}^{2 N+1}$.

The analogous scheme for the pseudospectral approximation was showned and analyzed in [F2]. Hence, stability and convergence for our approximation can be obtained by adapting the theory in [F2].

The purpose of this paper is to give preconditioning results for the linear system obtained from the proceeding schemes (1.3) and (1.4).

Multiplying both sides of the linear system $B_{N} U_{h}=F_{h}$ obtained from the scheme (1.3) and (1.4) by the weights matrix $W_{N}$, we have a symmetric linear system such that $\tilde{B}_{N} U_{h}:=W_{N} B_{N} U_{h}=W_{N} F_{h}$. It is well-known that the condition number of $\tilde{B}_{N}$ increase like $N^{2}$. Here, we shall study the preconditioning which is important for the sucessful application of conjugate gradient method.

As a preconditioner we take a stiffness matrix $\beta_{N}$ associated with the same Eq. (1.1) on the space $\mathcal{S}_{\Delta, 1}$ of the continuous piecewise linear functions 
which break at the collocation points. Now, we have a preconditioned linear system such that

$$
Q_{N} U_{h}:=\beta_{N}^{-1} \tilde{B}_{N} U_{h}=\beta_{N}^{-1} W_{N} F_{h}
$$

Following the ideas in [KP], we will show that the matrix $Q_{N}$ has all positive bounded eigenvalues independent of mesh size on a quasi-uniform mesh. In [KP], using Hermite cubic splines, Kim and Parter gave preconditioning results for the same problem (1.1) immediately, where they didn't collocate at boundary points. Indeed, our work is quite similar to the work in [KP] so that we will quote their results for the proofs of our results.

One major difference is that we handle the Neumann boundary conditions by a different way. The other difference is that we can directly derive one collocation matrix $B_{N}^{d}$ associated with Dirichlet boundary conditions, i.e., $u(0)=0, u(1)=0$, taking the interior $(2 N) \times(2 N)$ elements of $B_{N}$ and the other collocation matrix $B_{N}^{m}$ associated with mixed boundary conditions, i.e., $u(0)=0, u^{\prime}(1)=0$, eliminating the first row and the first column from $B_{N}$. Of course, we can obtain the preconditioning results for the Eq. (1.1) with Dirichlet boundary conditions or mixed boundary conditions using the matrix $B_{N}^{d}$ or $B_{N}^{m}$. Moreover, using the tensor products we can also extend the preconditioning strategy to a positive definite second order elliptic partial differential equation.

The rest of the paper is organized as follows. In section 2, we give some preliminary ideas, notations, etc. In section 3 , we will give one dimensional preconditioning result for the Neumann problem. And our discussions are extended to a positive definite second order elliptic partial differential equation with Neumann boundary conditions in section 4 . We give the computational results about one dimensional problem in section 5 .

\section{Preliminary}

Let $I$ be the unit interval and let $\Omega:=I \times I$ be the unit square. Let $\Delta$ be a partition of $I$ such as

$$
\Delta: 0=t_{0}<t_{1}<\cdots<t_{N}=1, \quad h_{i}:=t_{i}-t_{i-1}, \quad I_{i}=\left[t_{i-1}, t_{i}\right] .
$$

Let $h$ and $\rho$ be the mesh size and the bounded global mesh ratio of the partition $\Delta$, respectively, such that 


$$
h:=\max _{1 \leq i \leq N}\left\{h_{i}\right\} \quad \text { and } \quad 1 \leq \rho:=\max _{1 \leq i, j \leq N} \frac{h_{i}}{h_{j}} \leq \gamma .
$$

Throughout this paper, the work is based on the quasi-uniform mesh.

Using Legendre-Gauss points and Legendre-Gauss-Radau points (see [CHQZ]), we define the collocation points $\left\{\xi_{i}\right\}_{i=0}^{2 N+1}$ with the corresponding quadrature wights $\left\{w_{i}\right\}_{i=0}^{2 N+1}$ such as

$$
\left\{\begin{array} { r l } 
{ \xi _ { 0 } } & { = 0 } \\
{ \xi _ { 1 } } & { = \frac { h _ { 1 } } { 2 } ( \frac { 6 - \sqrt { 6 } } { 5 } ) } \\
{ \xi _ { 2 } } & { = \frac { h _ { 1 } } { 2 } ( \frac { 6 + \sqrt { 6 } } { 5 } ) } \\
{ \xi _ { 2 i - 1 } } & { = \frac { h _ { i } } { 2 } ( 1 - \frac { 1 } { \sqrt { 3 } } ) + t _ { i - 1 } } \\
{ \xi _ { 2 i } } & { = \frac { h _ { i } } { 2 } ( 1 + \frac { 1 } { \sqrt { 3 } } ) + t _ { i - 1 } } \\
{ \xi _ { 2 N - 1 } } & { = \frac { h _ { N } } { 2 } ( \frac { 4 - \sqrt { 6 } } { 5 } ) + t _ { N - 1 } } \\
{ \xi _ { 2 N } } & { = \frac { h _ { N } } { 2 } ( \frac { 4 + \sqrt { 6 } } { 5 } ) + t _ { N - 1 } } \\
{ \xi _ { 2 N + 1 } } & { = 1 }
\end{array} \left\{\begin{array}{rl}
w_{2} & =\frac{h_{1}}{2}\left(\frac{5^{3}(4+\sqrt{6})}{9(58+12 \sqrt{6})}\right) \\
w_{2} & =\frac{h_{i}}{2} \\
w_{2}(58-\sqrt{6}) & =\frac{h_{i}}{2} \\
w_{2 i} & w_{2 N-1}=\frac{h_{N}}{2}\left(\frac{5^{3}(4-\sqrt{6})}{9(58-12 \sqrt{6})}\right) \\
w_{2 N} & =\frac{h_{N}}{2}\left(\frac{5^{3}(4+\sqrt{6})}{9(58+12 \sqrt{6})}\right) \\
w_{2 N+1} & =\frac{h_{N}}{9} .
\end{array}\right.\right.
$$

Let $\mathcal{S}_{\Delta, 3}$ be the space of all $C^{1}$-cubic splines for the partition $\Delta$, i.e.,

$$
\mathcal{S}_{\Delta, 3}=\left\{u \in C^{1}[0,1]:\left.u\right|_{I_{i}} \text { is a cubic polynomial for each } i\right\}
$$

and let $\left\{\psi_{i}\right\}_{i=0}^{2 N+1}$ be the $C^{1}$-cubic Lagrange spline basis for the space $\mathcal{S}_{\Delta, 3}$ which satisfy $\psi_{i}\left(\xi_{j}\right)=\delta_{i, j}$ for $i, j=0,1, \ldots, 2 N+1$.

Let $\mathcal{S}_{\Delta, 1}$ be the space of continuous piecewise linear functions with basis $\left\{\phi_{i}\right\}_{i=0}^{2 N+1}$ which break at the collocation points $\left\{\xi_{i}\right\}_{i=0}^{2 N+1}$, i.e., $\phi_{i}\left(\xi_{j}\right)=\delta_{i, j}$.

Let $\pi:=\Delta \times \Delta$ be a partition of $\Omega$ and let us denote by two dimensional function spaces $\mathcal{S}_{\pi, 3}:=\mathcal{S}_{\Delta, 3} \otimes \mathcal{S}_{\Delta, 3}$ and $\mathcal{S}_{\pi, 1}:=\mathcal{S}_{\Delta, 1} \otimes \mathcal{S}_{\Delta, 1}$.

Let $(\cdot, \cdot)_{I}$ and $\|\cdot\|$ denote by the usual $L_{2}$ inner product and the corresponding norm:

$$
(u, v)_{I}=\int_{I} u v d t \quad \text { and } \quad\|u\|_{0}=\sqrt{(u, u)_{I}} .
$$


We will use the usual Sobolev norm. Let $H^{1}(I)$ be a Sobolev space

$$
H^{1}(I)=\left\{v \in L_{2}(I): v^{\prime} \in L_{2}(I)\right\} .
$$

The inner product and its associated norm on $H^{1}(I)$ are given by

$$
(u, v)_{H^{1}(I)}=\int_{I}\left(u^{\prime} v^{\prime}+u v\right) d t, \quad\|u\|_{1}=\sqrt{(u, u)_{H^{1}(I)}} .
$$

Define a quadratic form $[\cdot, \cdot]_{N}$ on $\mathcal{S}_{\Delta, 3} \times \mathcal{S}_{\Delta, 3}$ by

$$
[u, v]_{N}:=\sum_{i=0}^{2 N+1} w_{i} u\left(\xi_{i}\right) v\left(\xi_{i}\right) \quad \text { for } \quad u, v \in \mathcal{S}_{\Delta, 3} .
$$

Let us define the cubic spline interpolation operator $I_{N}$ as

$$
\left(I_{N} u\right)(t)=\sum_{i=0}^{2 N+1} u\left(\xi_{i}\right) \psi_{i}(t) \in \mathcal{S}_{\Delta, 3} \quad \text { for } \quad u \in \mathcal{S}_{\Delta, 1} .
$$

\section{Preconditioning on $1 \mathrm{D}$ case}

Theorem 3.1 For $\left\{\psi_{i}\right\}_{i=0}^{2 N+1}$, there exists a positive constant $C_{1}$, independent of mesh size, such that

$$
\max _{t_{j-1} \leq t \leq t_{j}}\left|\psi_{i}(t)\right| \leq C_{1}\left(\frac{1}{7}\right)^{\left|j-i^{*}\right|}
$$

where $i^{*}$ denotes the largest integer less than or equal to $(i+1) / 2$.

Proof. Using Theorem 1 in [KS], we can easily check that there is a positive constant $C_{1}$ which satisfies the estimate (3.1) for $\psi_{i}(i=1,2, \ldots, 2 N)$ and in addition, for $\psi_{0}$ and $\psi_{2 N+1}$.

Using Theorem 3.1 and repeating the appropriate modifications of the works in [KP], we have the following lemma:

Lemma 3.1 There exist positive constants $C_{2}, C_{3}$ and $C_{4}$, independent of mesh size, such that

$$
C_{2}\|u\|_{0}^{2} \leq[u, u]_{N} \leq C_{3}\|u\|_{0}^{2} \quad \text { for every } u \in \mathcal{S}_{\Delta, 3}
$$

and

$$
\left\|u^{\prime}\right\|_{0} \leq\left\|\left(I_{N} u\right)^{\prime}\right\|_{0} \leq C_{4}\left\|u^{\prime}\right\|_{0} \quad \text { for every } u \in \mathcal{S}_{\Delta, 1}
$$


Lemma 3.2 For every $u \in \mathcal{S}_{\Delta, 3}$, we have

$$
\left\|u^{\prime}\right\|_{0}^{2} \leq\left[-u^{\prime \prime}, u\right]_{N}+u^{\prime}(1) u(1)-u^{\prime}(0) u(0) \leq \frac{5}{3}\left\|u^{\prime}\right\|_{0}^{2} .
$$

Proof. Denote that $[f, g]_{I_{j}}$ and $(f, g)_{I_{j}}$ are the restrictions of $[f, g]_{N}$ and $(f, g)_{I}$ on $I_{j}$, respectively. Since $f^{\prime \prime} g$ is a polynomial of degree $\leq 4$ for $f, g$ $\in \mathcal{S}_{\Delta, 3}$ on subintervals $I_{1}$ and $I_{N}$, by Legendre-Gauss-Radau integration, we have

$$
\begin{aligned}
-\left[f^{\prime \prime}, g\right]_{I_{1}}+f^{\prime}\left(t_{1}\right) g\left(t_{1}\right)-f^{\prime}(0) g(0) & =\left(f^{\prime}, g^{\prime}\right)_{I_{1}}, \\
-\left[f^{\prime \prime}, g\right]_{I_{N}}+f^{\prime}(1) g(1)-f^{\prime}\left(t_{N-1}\right) g\left(t_{N-1}\right) & =\left(f^{\prime}, g^{\prime}\right)_{I_{N}} .
\end{aligned}
$$

By Lemma 3.1 in [CP] and [DD], there is a positive constant $P$ such that, for $k=2,3, \ldots, N-1$,

$$
\begin{array}{r}
-\left[f^{\prime \prime}, g\right]_{I_{k}}+f^{\prime}\left(t_{k}\right) g\left(t_{k}\right)-f^{\prime}\left(t_{k-1}\right) g\left(t_{k-1}\right) \\
=\left(f^{\prime}, g^{\prime}\right)_{I_{k}}+\frac{2}{3} P f_{k}^{(3)} g_{k}^{(3)} h_{k}^{5}
\end{array}
$$

where $f_{k}^{(3)}$ is the third derivative of $f$ on $I_{k}$.

Combining (3.5), (3.6) and (3.7), we have

$$
\begin{aligned}
-\left[f^{\prime \prime}, g\right]_{N}+f^{\prime}(1) & g(1)-f^{\prime}(0) g(0) \\
= & \left(f^{\prime}, g^{\prime}\right)_{I}+\frac{2}{3} P \sum_{k=2}^{N-1} f_{k}^{(3)} g_{k}^{(3)} h_{k}^{5} .
\end{aligned}
$$

From Lemma 3.2 in [DD], we observe that

$$
P \sum_{k=2}^{N-1} f_{k}^{(3)} g_{k}^{(3)} h_{k}^{5} \leq\left(f^{\prime}, g^{\prime}\right)_{I} .
$$

Setting $f=u$ and $g=u$ and combining (3.8) and (3.9), we have

$$
\left(u^{\prime}, u^{\prime}\right)_{I} \leq-\left[u^{\prime \prime}, u\right]_{N}+u^{\prime}(1) u(1)-u^{\prime}(0) u(0) \leq \frac{5}{3}\left(u^{\prime}, u^{\prime}\right)_{I}
$$

so it completes the proof.

Define two bilinear forms $a_{N}(\cdot, \cdot)$ and $b_{N}(\cdot, \cdot)$ on $\mathcal{S}_{\Delta, 3} \times \mathcal{S}_{\Delta, 3}$ as

$$
a_{N}(f, g)=f^{\prime}(1) g(1)-f^{\prime}(0) g(0):=\left.f^{\prime} g^{\prime}\right|_{0} ^{1},
$$




$$
b_{N}(f, g)=\left[-f^{\prime \prime}, g\right]_{N}+[c f, g]_{N}+a_{N}(f, g) .
$$

Using (3.8), we can rewrite the bilinear form $b_{N}$ as

$$
b_{N}(f, g)=\left(f^{\prime}, g^{\prime}\right)_{I}+[c f, g]_{N}+\frac{2}{3} P \sum_{i=2}^{N-1} f_{i}^{(3)} g_{i}^{(3)} h_{i}^{5}
$$

which implies that $b_{N}(\cdot, \cdot)$ is symmetric.

Theorem 3.2 For every $u \in \mathcal{S}_{\Delta, 1}$, there are positive constants $C_{5}, C_{6}$ and $C_{7}$, independent of mesh size, such that

$$
\left\|u^{\prime}\right\|_{0}^{2} \leq\left[-\left(I_{N} u\right)^{\prime \prime}, I_{N} u\right]_{N}+a_{N}\left(I_{N} u, I_{N} u\right) \leq C_{5}\left\|u^{\prime}\right\|_{0}^{2}
$$

and

$$
C_{6}\|u\|_{0}^{2} \leq\left[I_{N} u, I_{N} u\right]_{N} \leq C_{7}\|u\|_{0}^{2}
$$

Proof. From (3.4), we have

$$
\left\|\left(I_{N} u\right)^{\prime}\right\|_{0}^{2} \leq\left[-\left(I_{N} u\right)^{\prime \prime}, I_{N} u\right]_{N}+a_{N}\left(I_{N} u, I_{N} u\right) \leq \frac{5}{3}\left\|\left(I_{N} u\right)^{\prime}\right\|_{0}^{2} .
$$

Hence, by (3.3), we have one of the conclusion (3.13) with $C_{5}=5 C_{4} / 3$.

Once the estimate (3.2) is done, applying the arguments in Theorem 4.1 and Theorem 4.2 in [KP] word by word to a quasi-uniform mesh, we have another conclusion (3.14).

Theorem 3.3 For every $u \in \mathcal{S}_{\Delta, 1}$, there are positive constants $C_{8}$ and $C_{9}$, independent of mesh size, such that

$$
C_{8}\|u\|_{1}^{2} \leq b_{N}\left(I_{N} u, I_{N} u\right) \leq C_{9}\|u\|_{1}^{2} .
$$

Proof. Since $0<m \leq c(t) \leq M<\infty$, using(3.14) we have

$$
m C_{6}\|u\|_{0}^{2} \leq\left[c I_{N} u, I_{N} u\right]_{N} \leq M C_{7}\|u\|_{0}^{2} .
$$

Now, combining (3.13) and (3.16) we have the conclusion (3.15).

With

$$
u=\sum_{j=0}^{2 N+1} u\left(\xi_{j}\right) \psi_{j} \in \mathcal{S}_{\Delta, 3},
$$


we collocate the Eq. (1.1) at the interior collocation points $\xi_{i}(i=1,2, \ldots, 2 N)$ such as

$$
\sum_{j=0}^{2 N+1}\left[-u\left(\xi_{j}\right) \psi_{j}^{\prime \prime}\left(\xi_{i}\right)+c\left(\xi_{j}\right) u\left(\xi_{i}\right) \psi_{j}\left(\xi_{i}\right)\right]=f\left(\xi_{i}\right)
$$

To impose boundary conditions, we can choice the following conditions

$$
\begin{aligned}
-u^{\prime \prime}(0)+c(0) u(0)-w_{0}^{-1} u^{\prime}(0) & =f(0) \\
-u^{\prime \prime}(1)+c(1) u(1)+w_{2 N+1}^{-1} u^{\prime}(1) & =f(1) .
\end{aligned}
$$

Then, combining the schemes (3.17) and (3.18) we have the collocation matrix $B_{N}$ for the Eq. (1.1) with the Neumann boundary conditions (1.2) such that

$$
B_{N} U_{h}=F_{h}
$$

where $U_{h}=\left(u\left(\xi_{0}\right), u\left(\xi_{1}\right), \ldots, u\left(\xi_{2 N+1}\right)\right)^{t}, F_{h}=\left(f\left(\xi_{0}\right), f\left(\xi_{1}\right), \ldots, f\left(\xi_{2 N+1}\right)\right)^{t}$ and the elements of $B_{N}$ are represented as

$$
\begin{aligned}
B_{N}(0, j) & =-\psi_{j}^{\prime \prime}(0)+c(0) \delta_{0, j}-w_{0}^{-1} \psi_{j}^{\prime}(0) \\
B_{N}(i, j) & =-\psi_{j}^{\prime \prime}\left(\xi_{i}\right)+c\left(\xi_{i}\right) \delta_{i, j} \\
B_{N}(2 N+1, j) & =-\psi_{j}^{\prime \prime}(1)+c(1) \delta_{2 N+1, j}+w_{2 N+1}^{-1} \psi_{j}^{\prime}(1)
\end{aligned}
$$

for $i=1,2 \cdots, 2 N$ and $j=0,1, \ldots, 2 N+1$.

Let $\tilde{B}_{N}:=W_{N} B_{N}$ be the symmetric matrix where $W_{N}$ is a weights matrix. Comparing $\tilde{B}_{N}(i, j)$ with $b_{N}\left(\psi_{j}, \psi_{i}\right)$ in (3.11) we have

$$
\tilde{B}_{N}(i, j)=\left[W_{N} B_{N}\right](i, j)=b_{N}\left(\psi_{j}, \psi_{i}\right)
$$

so that $\tilde{B}_{N}$ is symmetric.

Consider the stiffness matrix $\beta_{N}$ associated with the finite element discretization of the Eq. (1.1) in the space $\mathcal{S}_{\Delta, 1}$ as preconditioner:

$$
\beta_{N}(i, j)=\left(\phi_{i}^{\prime}, \phi_{j}^{\prime}\right)_{I}+\left(c \phi_{i}, \phi_{j}\right)_{I}
$$

The condition $m \leq c(t) \leq M$ for $t \in I$ implies that $U_{h}^{t} \beta_{N} U_{h}$ is equivalent to $\|u\|_{1}^{2}$ in $\mathcal{S}_{\Delta, 1}$, in the sense that

$$
\begin{aligned}
\min \{1, m\}\|u\|_{1}^{2} & \leq U_{h}^{t} \beta_{N} U_{h} \\
& \leq \max \{1, M\}\|u\|_{1}^{2} \quad \text { for } \quad u \in \mathcal{S}_{\Delta, 1} .
\end{aligned}
$$


Multiplying $\tilde{B}_{N}$ by $\beta_{N}^{-1}$, we have a preconditioned linear system such that

$$
Q_{N} U_{h}:=\beta_{N}^{-1} \tilde{B}_{N} U_{h}=\beta_{N}^{-1} W_{N} F_{N} .
$$

Now we will prove the main results that the preconditioned matrix $Q_{N}$ has all positive bounded eigenvalues, independent of mesh size.

Theorem 3.4 For every $u \in \mathcal{S}_{\Delta, 1}$, there are two positive constants $C_{10}$ and $C_{11}$, independent of mesh size, such that

$$
C_{10}\left(U_{h}^{t} \beta_{N} U_{h}\right) \leq U_{h}^{t} \tilde{B}_{N} U_{h} \leq C_{11}\left(U_{h}^{t} \beta_{N} U_{h}\right)
$$

with $U_{h}=\left(u\left(\xi_{0}\right), u\left(\xi_{1}\right), \ldots, u\left(\xi_{2 N+1}\right)\right)^{t}$.

Moreover the eigenvalues $\left\{\lambda_{j}\right\}_{j=0}^{2 N+1}$ of the matrix $Q_{N}:=\left(\beta_{N}\right)^{-1} \tilde{B}_{N}$ satisfy

$$
C_{10} \leq \lambda_{j} \leq C_{11} \quad \text { for } j=0,1, \ldots, 2 N+1 .
$$

Proof. Since $U_{h}$ represents both $u$ and $I_{N} u$, we have

$$
\begin{aligned}
b_{N}\left(I_{N} u, I_{N} u\right) & =\sum_{i, j} u\left(\xi_{i}\right) u\left(\xi_{j}\right) b_{N}\left(\psi_{i}, \psi_{j}\right) \\
& =\sum_{i, j} u\left(\xi_{i}\right) u\left(\xi_{j}\right) \tilde{B}_{N}(i, j)=U_{h}^{t} \tilde{B}_{N} U_{h} .
\end{aligned}
$$

By (3.15), we have

$$
C_{8}\|u\|_{1}^{2} \leq U_{h}^{t} \tilde{B}_{N} U_{h} \leq C_{9}\|u\|_{1}^{2} .
$$

Combining this estimate and (3.21), we have one of the conclusions (3.23). Now if $\lambda$ is one of the eigenvalues of $Q_{N}$ and $U_{h}$ is the corresponding eigenvector, then

$$
Q_{N} U_{h}=\left(\beta_{N}\right)^{-1} \tilde{B}_{N} U_{h}=\lambda U_{h} \quad \text { or } \quad \tilde{B}_{N} U_{h}=\lambda \beta_{N} U_{h} .
$$

Hence, we have $U_{h}^{t} \tilde{B}_{N} U_{h}=\lambda U_{h}^{t} \beta_{N} U_{h}$ which implies the other conclusion (3.24).

Remark. From the linear system of order $(2 N+2) \times(2 N+2)$ for the Eq. (1.1) with a Neumann boundary conditions (1.2), we can directly derive the two linear systems for the same Eq. (1.1) with Dirichlet boundary conditions $u(0)=0, u(1)=0$ and mixed boundary conditions $u(0)=0$, $u^{\prime}(1)=0$, and we can also investigate preconditioning results for these problems by a similar way as the case of Neumann problem. 
1) For the case of Dirichlet boundary value problem, we have a linear system of order $(2 N) \times(2 N)$ such as

$$
B_{N}^{d} U_{h}^{d}=F_{h}^{d} \quad \text { or } \quad\left(\beta_{N}^{d}\right)^{-1} W_{N}^{d} B_{N}^{d} U_{h}^{d}=\left(\beta_{N}^{d}\right)^{-1} W_{N}^{d} F_{h}^{d}
$$

where $B_{N}^{d}, W_{N}^{d}$ and $\beta_{N}^{d}$ are $(2 N) \times(2 N)$ matrices whose elements are given by

$$
\begin{gathered}
B_{N}^{d}(i, j)=B_{N}(i, j), \quad W_{N}^{d}=W_{N}(i, j), \quad \beta_{N}^{d}(i, j)=\beta_{N}(i, j), \\
(i, j=1,2, \ldots, 2 N),
\end{gathered}
$$

and $U_{h}^{d}:=\left(u\left(\xi_{1}\right), u\left(\xi_{2}\right), \ldots, u\left(\xi_{2 N}\right)\right)^{t}, F_{h}^{d}:=\left(f\left(\xi_{1}\right), f\left(\xi_{2}\right), \ldots, f\left(\xi_{2 N}\right)\right)^{t}$.

2) For the case of mixed boundary value problem, we have a linear system of order $(2 N+1) \times(2 N+1)$ such as

$$
B_{N}^{m} U_{h}^{m}=F_{h}^{m} \quad \text { or } \quad\left(\beta_{N}^{m}\right)^{-1} W_{N}^{m} B_{N}^{m} U_{h}^{m}=\left(\beta_{N}^{m}\right)^{-1} W_{N}^{m} F_{h}^{m}
$$

where $B_{N}^{m}, W_{N}^{m}$ and $\beta_{N}^{m}$ are $(2 N+1) \times(2 N+1)$ matrices whose elements are given by

$$
\begin{gathered}
B_{N}^{m}(i, j)=B_{N}(i, j), \quad W_{N}^{m}=W_{N}(i, j), \quad \beta_{N}^{m}(i, j)=\beta_{N}(i, j), \\
(i, j=1,2, \ldots, 2 N+1),
\end{gathered}
$$

and $U_{h}^{m}:=\left(u\left(\xi_{1}\right), u\left(\xi_{2}\right), \ldots, u\left(\xi_{2 N+1}\right)\right)^{t}, F_{h}^{m}:=\left(f\left(\xi_{1}\right), f\left(\xi_{2}\right), \ldots, f\left(\xi_{2 N+1}\right)\right)^{t}$.

\section{Preconditioning on 2D case}

Let $L_{\pi}$ be a differential operator such that

$$
L_{\pi}:=-\Delta u+\left(c_{1}(x)+c_{2}(y)\right) u \text { in } \Omega
$$

with Neumann boundary condition

$$
\frac{\partial u}{\partial \mathbf{n}}=0 \quad \text { on } \quad \partial \Omega
$$

where $\partial \Omega$ is the boundary of $\Omega, \frac{\partial u}{\partial \mathbf{n}}$ denotes the outward normal derivative of $u$ on $\partial \Omega$ and, $c_{1}$ and $c_{2}$ are continuous functions such that

$$
0<m \leq c_{1}(x), c_{2}(y) \leq M<\infty \text { for } 0 \leq x, y \leq 1 .
$$

Let us decompose the operator $L_{\pi}:=L_{x}+L_{y}$ such that

$$
\begin{aligned}
& \left(L_{x} u\right)(x, y)=-u_{x x}(x, y)+c_{1}(x) u(x, y), \\
& \left(L_{y} u\right)(x, y)=-u_{y y}(x, y)+c_{2}(y) u(x, y) .
\end{aligned}
$$


Ordering the collocation points $P_{\mu}:=\left(\xi_{i}, \xi_{j}\right)(\mu=j+i(2 N+2))$ and following the ideas in (3.19), we can obtain the symmetric matrix representations $\tilde{B}_{N_{x}} \otimes W_{N}$ and $W_{N} \otimes \tilde{B}_{N_{y}}$ of the cubic spline collocation discretization of $L_{x}$ and $L_{y}$, respectively (see [PR] and [WM]). Combining these matrices we have the symmetric matrix representation $\tilde{B}_{N^{2}}$ of the cubic spline collocation discretization of $L_{\pi}$ in the space $\mathcal{S}_{\pi, 3}$ such that

$$
\tilde{B}_{N^{2}}=\tilde{B}_{N_{x}} \otimes W_{N}+W_{N} \otimes \tilde{B}_{N_{y}} .
$$

Furthermore we have the stiffness matrix $\beta_{N^{2}}$ associated with the finite element discretization of $L_{\pi}$ in the space $\mathcal{S}_{\pi, 1}$ such that

$$
\beta_{N^{2}}=\beta_{N_{x}} \otimes M_{N}+M_{N} \otimes \beta_{N_{y}}
$$

where $M_{N}(i, j)=\left(\phi_{i}, \phi_{j}\right)_{I}$ and

$$
\begin{aligned}
& \beta_{N_{x}}(i, j)=\left(\phi_{i}^{\prime}, \phi_{j}^{\prime}\right)_{I}+\left(c_{1} \phi_{i}, \phi_{j}\right)_{I}, \\
& \beta_{N_{y}}(i, j)=\left(\phi_{i}^{\prime}, \phi_{j}^{\prime}\right)_{I}+\left(c_{2} \phi_{i}, \phi_{j}\right)_{I} .
\end{aligned}
$$

Note that $\tilde{B}_{N^{2}}$ and $\beta_{N^{2}}$ are symmetric and positive definite.

Lemma 4.1 There are positive constants $T_{1}$ and $T_{2}$, independent of mesh size, such that for all nonzero $U_{\pi} \in \mathbb{R}^{(2 N+2)^{2}}$

$$
T_{1} \leq \frac{U_{\pi}^{t}\left(\tilde{B}_{N_{x}} \otimes W_{N}\right) U_{\pi}}{U_{\pi}^{t}\left(\beta_{N_{x}} \otimes M_{N}\right) U_{\pi}} \leq T_{2}
$$

and

$$
T_{1} \leq \frac{U_{\pi}^{t}\left(W_{N} \otimes \tilde{B}_{N_{y}}\right) U_{\pi}}{U_{\pi}^{t}\left(M_{N} \otimes \beta_{N_{y}}\right) U_{\pi}} \leq T_{2} .
$$

Proof. Observe that

$$
\left(\beta_{N_{x}} \otimes M_{N}\right)^{-1}\left(\tilde{B}_{N_{x}} \otimes W_{N}\right)=\beta_{N_{x}}^{-1} \tilde{B}_{N_{x}} \otimes M_{N}^{-1} W_{N} .
$$

By Theorem 3.4 all eigenvalues $\lambda_{j}$ of $\beta_{N_{x}}^{-1} \tilde{B}_{N_{x}}$ satisfy $C_{10} \leq \lambda_{j} \leq C_{11}$. By (3.14) in Theorem 3.2 we can show that all eigenvalues $\mu_{j}$ of $M_{N}^{-1} W_{N}$ satisfy $C_{6} \leq \mu_{j} \leq C_{7}$. Therefore the eigenvalues $\nu_{j}=\lambda_{j} \mu_{j}$ of (4.6) satisfy $C_{10} C_{6} \leq$ $\nu_{j} \leq C_{11} C_{7}$ where the constants $C_{6}, C_{7}, C_{10}$ and $C_{11}$ are independent of mesh size.

Hence we have (4.4). Also, we can obtain the conclusion (4.5) by a similar way. 
Theorem 4.1 Let $Q_{N^{2}}:=\beta_{N^{2}}^{-1} \tilde{B}_{N^{2}}$. Then we have the following main results:

For all nonzero $U_{\pi} \in \mathbb{R}^{(2 N+2)^{2}}$,

$$
T_{1} \leq \frac{U_{\pi}^{t} \tilde{B}_{N^{2}} U_{\pi}}{U_{\pi}^{t} \beta_{N^{2}} U_{\pi}} \leq T_{2}
$$

Furthermore, the eigenvalues $\left\{\Lambda_{\mu}\right\}$ of the matrix $Q_{N^{2}}$ satisfy

$$
T_{1} \leq \Lambda_{\mu} \leq T_{2}
$$

Proof. From (4.2) and (4.3), we can see that

$$
U_{\pi}^{t} \tilde{B}_{N^{2}} U_{\pi}=U_{\pi}^{t}\left(\tilde{B}_{N_{x}} \otimes W_{N}\right) U_{\pi}+U_{\pi}^{t}\left(W_{N} \otimes \tilde{B}_{N_{y}}\right) U_{\pi}
$$

and

$$
U_{\pi}^{t} \beta_{N^{2}} U_{\pi}=U_{\pi}^{t}\left(\beta_{N_{x}} \otimes M_{N}\right) U_{\pi}+U_{\pi}^{t}\left(M_{N} \otimes \beta_{N_{y}}\right) U_{\pi} .
$$

Using (4.4) and (4.5), we can easily obtain that

$$
T_{1} U_{\pi}^{t} \beta_{N^{2}} U_{\pi} \leq U_{\pi}^{t} \tilde{B}_{N^{2}} U_{\pi} \leq T_{2} U_{\pi}^{t} \beta_{N^{2}} U_{\pi} .
$$

This estimate completes the conclusion (4.7) which implies (4.8).

\section{Computational Results}

Consider the following boundary-value problem:

$$
\begin{aligned}
& L u:=-u^{\prime \prime}+u=f \quad \text { in } I \\
& u^{\prime}(0)=0 \quad \text { and } \quad u^{\prime}(1)=0 .
\end{aligned}
$$

From (3.19), we have the symmetric matrix representation $\tilde{B}_{N}$ for the Eq. (5.1) with the Neumann boundary conditions (5.2) such that

$$
\tilde{B}_{N} U_{h}:=W_{N} B_{N} U_{h}=W_{N} F_{h}
$$

where $U_{h}=\left(u\left(\xi_{i}\right)\right)^{t}$ and $F_{h}=\left(f\left(\xi_{i}\right)\right)^{t}$.

From (3.22), we have preconditioned algebraic linear system:

$$
Q_{N} U_{N}:=\beta_{N}^{-1} W_{N} B_{N} U_{N}=\beta_{N}^{-1} W_{N} F_{N} .
$$

For $\tilde{B}_{N}$, we have all positive real eigenvalues epending on $N$. Unfortunately, 
the condition number

$$
k\left(\tilde{B}_{N}\right):=\frac{\max \left\{\text { Eigenvalues of } \tilde{B}_{N}\right\}}{\min \left\{\text { Eigenvalues of } \tilde{B}_{N}\right\}}
$$

increases like $N^{2}$. This results in a very slow convergence by iterative method (e.g., Conjugate Gradient method and Richardson method).

For $Q_{N}$ based on the quasi uniform mesh, we have all positive bounded eigenvalues independent of $N$. Hence the condition number $k\left(Q_{N}\right)$ of $Q_{N}$ turns out to be bounded, independent of $N$.

\begin{tabular}{|c|c|c|}
\hline $\mathrm{N}$ & $\tilde{B}_{N}$ & $Q_{N}$ \\
\hline 8 & 2812 & 2.163556 \\
\hline 16 & 11243 & 2.187724 \\
\hline 32 & 44969 & 2.194010 \\
\hline 64 & 179876 & 2.195612 \\
\hline 128 & 719500 & 2.196017 \\
\hline 256 & 2877997 & 2.196118 \\
\hline
\end{tabular}

Table 1. Condition numbers of $\tilde{B}_{N}$ and $Q_{N}$

In Table 1, we report the condition numbers of $\tilde{B}_{N}$ and $Q_{N}$, respectively, where the work is based on the uniform mesh (i.e., $h=h_{i}$ for all $i$ ).

\section{References}

[BF] Bertoluzza S. and Funaro D., Remarks about the Matrices relative to the Psedospectral Approximation of Neumann Problems. Proceedings of the IMACS (International Symposium on Iterative Methods), Bruxells, 2/4 april 1991 (R. Beauwens \& P. de Groen Eds., North Holland. Amsterdam 1992), 209-215.

[CHQZ] Canuto C., Hussaini M. Y., Quarteroni A. and Zang T.A., Spectral Methods in Fluid Dynamics, Springer Series in Computational Physics. Springer-Verlag, Berlin, New York, (1988).

[CP] Cerutti J. and Parter S.V., Collocation methods for Parabolic Partial Differential Equations in one dimensional space. Numer. Math. 26 (1974), 227-254.

[DD] Douglas J. and Dupont T., Collocation Methods for Parabolic Equations in a Single Space Variable. Lecture Notes in Mathematics, 385, Springer-Verlag, (1974).

[F1] Funaro D., A multidomain spectral approximation of elliptic equations. Numerical Methods for P.D.E. 2 (1986), 187-205. 
[F2] Funaro D., Domain Decomposition Methods for Pseudo Spectral Approximations. Numer. Math. 58 (1988), 329-344.

[KK] Kim S.D. and Kim S.C., Exponential decay of $C^{1}$-cubic splines vanishing at the local interior symmetric points. (accepted to Numer. Math.).

[KP] Kim S.D. and Parter S. V., Preconditioning Cubic Spline Collocation Discretizations of Elliptic Equations. Numer. Math. 72 (1995), 39-72.

[KS] Kim S.D. and Shin B.C., On $C^{1}$ cubic Lagrange splines vanishing at local unsymmetric interior points in a nonuniform mesh. (submit to Houston Jour. of Math.).

[O] Orszag S.A., Spectral methods for problems in complex geometries. J. Comput. Phys. 37 (1980), 70-92.

[PR] Parter S.V. and Rothman S. P., Preconditioning second-order elliptic operators: Condition numbers and the distribution of the singular values. Journal of Scientific Computation, 6 (1991), 1927-1957.

[WM] Wait R. and Mitchell A.R., Finite Element Analysis and Applications. John Wiley and Sons, (1995).

Sang Hun Lee

Department of Mathematics

College of Natural Sciences

Kyungpook National University

Taegu, 702-701, Korea

Tel. 950-5310, Fax. 950-6306

E-mail: sanghlee@bh.kyungpook.ac.kr

Byeong Chun Shin

Department of Mathematics

College of Natural Sciences

Kyungpook National University

Taegu, 702-701, Korea

Tel. 950-5310, Fax. 950-6306

E-mail: cshin@gauss.kyungpook.ac.kr 